\title{
Masseter muscle adaptation following orthognathic surgery - Biomodelling analysis - A pilot study
}

Duarte $F^{1}$, Silva $\mathrm{JN}^{2}$, Hopper $\mathrm{C}^{3}$, Hunt $\mathrm{N}^{4}$

Affiliations:

1. Oral Surgeon Specialist by OMD (Portuguese Dental Association)

Post-Graduation in Oral and Maxillofacial

Surgery at Eastman Dental Institute - University College of London

Master of Science in Oral and Maxillofacial

Surgery at Eastman Dental Institute - University College of London

PhD student at UCL - University College of

London

Professor at ISAVE - Instituto Superior de Saúde - Portugal

CEO and Clinical Director of Clitrofa - Trofa - Portugal

2.Professor at ISAVE - Instituto Superior de Saúde - Portugal

Member of the Interdisciplinary Center for

Health Sciences (ICHS) - ISAVE -Instituto Superior de Saúde

3.UCL Eastman Dental Institute, Oral \&

Maxillofacial Surgery Department

4.UCL Eastman Dental Institute, Orthodontics Department

Corresponding author:

Fernando Duarte

Clitrofa - Centro Médico, Dentário e Cirúrgico

fduarte@clitrofa.com

\section{ABSTRACT}

Purpose: This pilot investigation was designed to apply several, newly developed and more sophisticated methods of measuring muscle structure, function and fibre orientation to a situation where adaptation of muscle is pivotal to the success of a therapeutic approach.

Materials and Methods: Patients attending the combined orthodontic / orthognathic surgery clinic at Clitrofa - Centro Médico, Dentário e Cirúrgico, in Trofa - Portugal were screened using Magnetic Resonance Imaging protocol. Ten patients scheduled for a bimaxillary osteotomy involving a combination of maxillary Le Fort I impaction procedure coupled with a sagittal split advancement of the mandible were select to form the study group. An Experimental design used to provide information in relation to masticatory muscle adaptation following orthognathic surgery. The study involved the contribution of two independent examiners that measured the changes in fibre orientation at the different jaw positions using Anatomics ${ }^{\top \mathrm{M}}$ software, at three different time moments. A combination of different parametric tests has been used to compare the different experimental variables.

Results: Statistical differences have been identified between examiners measurements and between operations. There were no significant differences testing different times. Conclusions: The discrepancies between examiners probably arise from small variations in the experimental methodology used by them. The differences between operations reveal masseter muscle adaptation following orthognathic surgery. The measurement of "P1 masseter muscle/ zygomatic bone / process mastoid anterior angle" and "P2 masseter muscle / mandibular angle" can therefore be a valuable tool for controlling the reworking of masseter muscle upon orthognathic surgery.

\section{KEYWORDS}

Orthognathic surgery, masseter muscle, biomodelling analysis 


\section{INTRODUCTION}

Orthognathic surgery is a practical art, the surgeon often uses direct physical intervention in the treatment of patients. To minimize operative morbidity and mortality, and to maximize therapeutic success, surgical strategies are tailored to each patient and must be carefully planned to use the best possible anatomical information. The traditional way for a surgeon to gain basic experience without risk to the patient is to dissect cadavers and to examine carefully preserved pathological specimens. This serves to provide a conceptual anatomopathological framework from which operative interventions may be safely made. However, every patient is unique. Thus, there is a need for the surgeon to attain a specific understanding of the individual's anatomy pre-operatively. Thorough physical examination may be all that is needed for conditions in which the anatomopathology is common and the surgeon experienced. With complicated anatomopathology, detailed information relating to the morphology of internal structures is often required by the surgeon to enhance understanding. To obtain this internal anatomical information non-invasively, the surgeon relies on medical imaging. ${ }^{1}$

Advances in medical imaging have created ever increasing volumes of complex data obtained from the patient. The interpretation of such information has become a specialty in itself and the surgeon at times may be left bewildered as to how best to apply the available information to the practicalities of physical intervention. The surgeon seeks to understand the exact morphology of the abnormality, its relationships to the surrounding anatomy and the best way to access and correct the pathology operatively. Such specific information is not readily available in the radiologist's report and however experienced the surgeon may be at interpreting images such questions often cannot be easily answered. ${ }^{1}$

Three-dimensional (3D) imaging has been developed to narrow the communication gap between radiologist and surgeon. By using 3D imaging a vast number of complex slice images can be quickly appreciated. The term "three-dimensional" however, is not a truly accurate description of these images as they are still displayed in a radiological film or flat screen in only two dimensions1. The advent of 3D imaging has not only improved data display, but also promoted the development of even more useful technologies to assist the surgeon in the diagnosis and planning. ${ }^{1}$

For harmonious vertical facial growth and development to exist, the growth on the front of the face must be the same as on the back. If this does not occur, there may be a relative growth rotation of the mandible. For example, if the growth in the posterior part of the face exceeds what occurred previously, the net effect will be an anterior rotation of the mandible, producing the typical deformity of the short face and the deep overbite associated with the short face syndrome. ${ }^{2}$ At the opposite end, where growth at the back of the face can be severely reduced compared to what occurred earlier, a clockwise opening or rotation of the jaw is evident, with the net effect of being an excessive anterior facial height and often a bitten anterior opening, associated with a deformity of the long face. ${ }^{3}$

For generations, both clinicians and scientists have argued as to the respective contribution of genetics and, so called, environmental factors in influencing ultimated facial form and associated malocclusion. Of all the possible environmental influences, it is not surprising that bearing in mind the origins and insertions of the muscles of mastication, and in particular the masseter and medial pterygoid muscle, that the question has arisen as to whether or not abnormalities in the structure and function of the muscular pterygomasseteric sling (PMS) could, in any way, influence vertical development in the posterior part of the face. Furthermore, if treatment interventions necessitate a change in function of the muscles that support the mandible, do the adaptive capability of these muscles in any way influence the stability of the treatment outcome. ${ }^{4}$

\section{BIOMODELLING}

Biomodelling is the generic term describing the ability to replicate the morphology of a biological structure in a solid substance. Specifically, biomodelling has been defined as "the process of using radiant energy to capture morphological data on a biological structure and the processing of such data by a computer to generate the code required to manufacture the structure by rapid prototyping apparatus". A biomodel is the product of this process, and virtual reality is the generic term coined for the visualization medium. ${ }^{1}$

Computers are used increasingly as a supportive tool for the diagnosis, operation planning, and treatment in medicine and dentistry. They are used in connection with the modern digital imaging techniques such as computer tomography and magnetic resonance imaging, as well as ultrasound to improve the visualization of anatomical and physiological conditions in keeping with the human imagination. ${ }^{5}$

The ability to extract accurate three-dimensional (3D) images from MRI, has proven to be a very useful diagnostic tool, using a standardize scanning process, with fine overlapping slices of $1 \mathrm{~mm}$ thickness and a spacing of $0.8 \mathrm{~mm}$ during 7 minutes, was possible to extract the muscles and the facial bones from same scan. ${ }^{6}$

The objective was to extract the muscle from the scan with secure margins identification and also to extract the facial bones with considerable detail. The software used was the Anatomics ${ }^{\mathrm{TM}}$ that allows the correction of muscle and bone limits at any time. The reconstruction of muscles and bone from the same scan has allowed visualisation of the muscle fibre orientation in relation to the muscle's bony attachments. This could enable the measurement of potential changes in orientation in relation to a static landmark unaffected by surgery (e.g. Frankfort plane) or in relation to functional identifiers (e.g. Occlusal plane).

\section{MUSCLES ROLE}

Many forms of interceptive treatment, whether they be purely orthodontic in nature or in combination with surgery, bring about changes in the muscles of mastication with regard to one or more of the following changes: a) in muscle fibre orientation, b) changes in the functioning length of fibres, c) changes in muscle structure and d) changes in muscle phenotype. Successful treatment requires both reorganization in the connective tissue and regeneration of muscle fibres. Reorganization of connective tissue is an extremely complex process involving muscle derived stem cells (satellite cells), extra-cellular matrix molecules and receptors for the extra-cellular matrix (for example integrins). Remodeling of the extra-cellular matrix is mediated by a family of enzymes known as matrix metalloproteinases (MMPs). 7,8 MMP2 is expressed during the regeneration of new myofibres and is a known mechano-responsive gene. A knowledge of how muscles respond to clinical interventions is pivotal to treatment success and can influence the way in which a particular treatment modality is applied. Functional appliances, for example, can be either fixed or removable, can be constructed to varying degrees of vertical opening and there are protagonists and antagonists for both gradual versus one-step activation of the appliances. Similarly, distraction osteogenesis is considered by many to be preferable to orthognathic surgery in specific cases because it induces a gradual as opposed to a one-step activation believed 
to be more physiologically appropriate for bone and possibly, muscle adaptation. ${ }^{7,8}$

With regard to orthognathic surgery the golden rule is that surgery must not stretch the pterygomasseteric sling, otherwise relapse is likely to occur. This is predominantly through the speed of insult to the muscle in relation to the timing of the muscle adaptive process. The consequence is either an immediate reversion back to the original functioning length of the muscle and return of the bony fragments back to their original presurgical position, and/or migration of the muscle attachment along the surface of the bone, thereby leading to an area of bone denuded of muscle force, which ultimately leads to resorption of the bony muscular processes.

One way in which this can be studied more closely is through refinements in protocols for $3 \mathrm{D}$ magnetic resonance imaging of the face and jaws. Increasing the resolution of the tomographic cuts to $1.0 \mathrm{~mm}$ has led to a resolution which facilitates the identification of not only the origins and insertions of the muscles of mastication but even the orientation of individual muscle fibre bundles (Figure $1 \mathrm{~A}$ and $\mathrm{B}$ ). It is therefore possible to study the changes in muscle fibre orientation in relation to landmarks such as the functional occlusal plane and also those landmarks unaffected by surgery, for example the cranial base (Figure 1C and D). Ideally, as mentioned, surgery to correct an increased vertical facial deformity should involve posterior maxillary impaction together with a mandibular procedure where the final outcome does not increase the posterior facial height and hence, does not stretch the pterygomasseteric sling. As such the orientation of the muscle fibres in relation to their functioning occlusal plane remains unaltered (Figure 1E). However, if there is failure to adequately impact the posterior part of the maxilla in such cases, then there is a rotation of the mandibular segments around the premolar/first molar region, resulting in a reduction of the anterior face height but, an unwelcome increase in the posterior vertical dimension (Figure 1 F) and thereby leading to an increase in the length of the pterygomasseteric sling (Figure $1 \mathrm{G})$. Furthermore, this leads to a much less efficient musculoocclusal relationship and as such more extensive adaptation must take place within the muscles in order to be able to accommodate the unwanted surgical change. In clinical cases where this unwanted change has occurred, there is not only a return towards the original pre-surgical bony relationships (Figure $1 \mathrm{H}$ ) but also migration of the muscle attachment leaving an area of bone at the gonial angle which subsequently resorbs and leads to the unwanted and unsightly hour glass deformity of the mandibular border (Figure 1I).
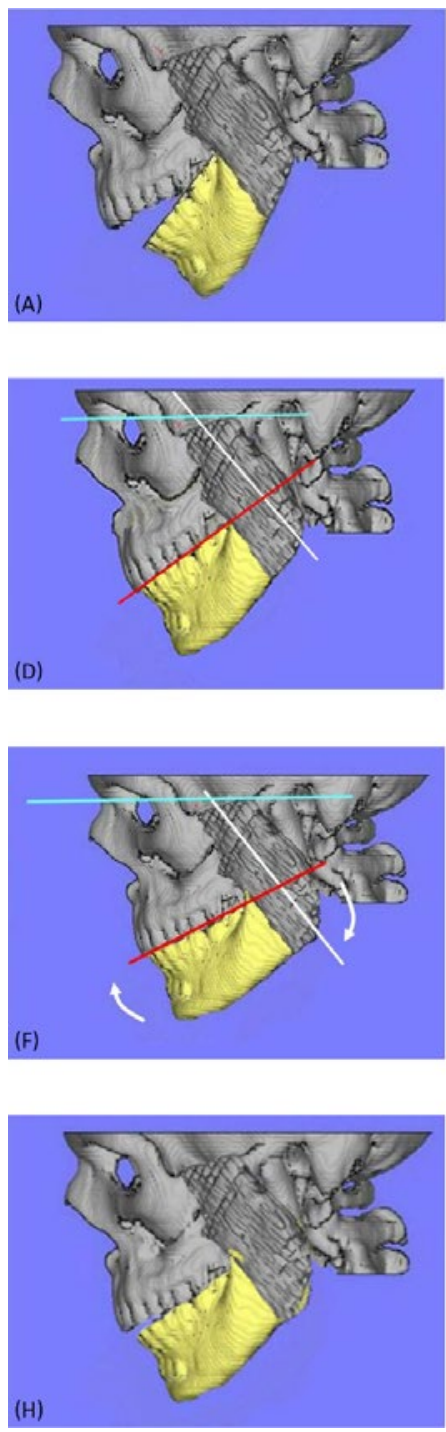
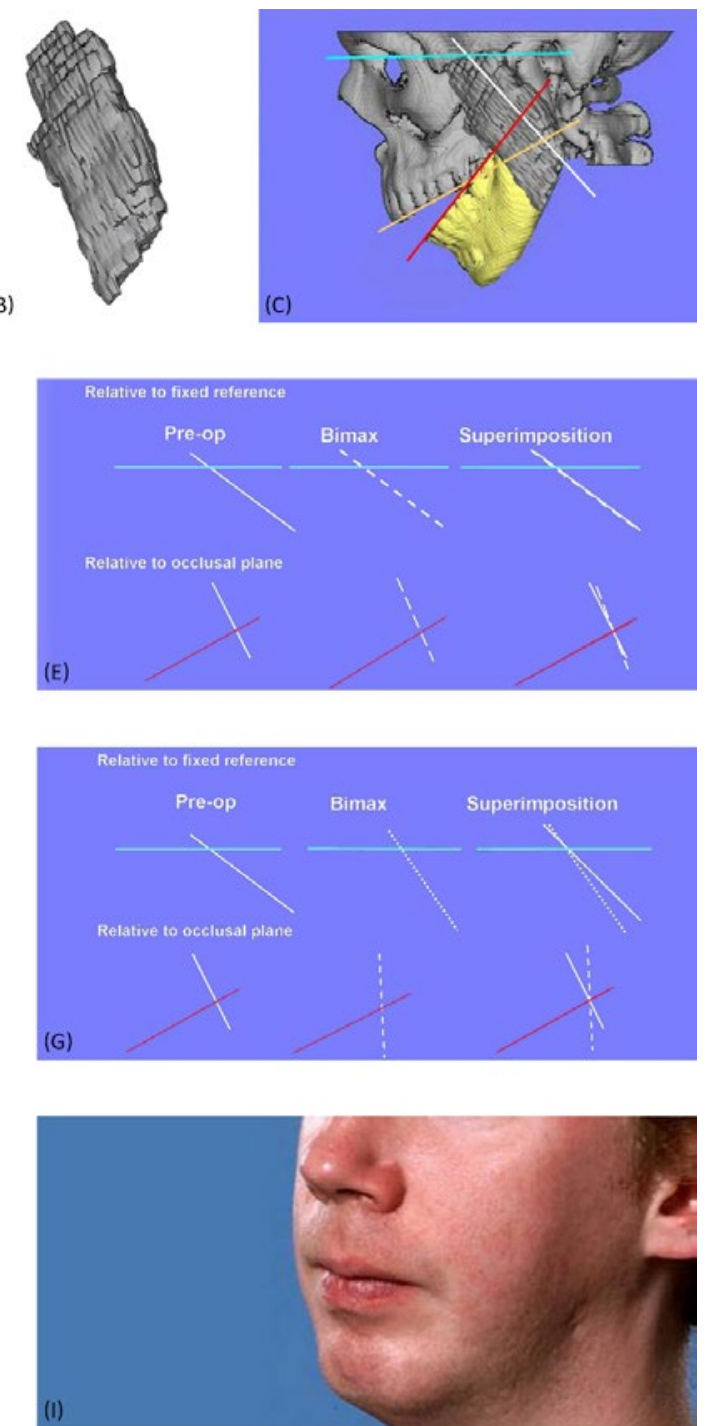

Figure 1. 3D MRI (Magnetic Resonance Imaging) shows detail of masseter muscle fibre bundle orientation (A and B). Favorable change in muscle length and fibre orientation following maxillary impaction and mandibular advancement surgery for closure of anterior open bite (C, D and E). Unfavorable change following insufficient posterior maxillary impaction with resultant stretch of pterygomandibular sling ( $\mathrm{F}$ and $\mathrm{G})$ and subsequent relapse ( $\mathrm{H}$ and $\mathrm{I})$. 


\section{MATERIALS AND METHODS}

Patients attending the combined orthodontic/orthognathic surgery clinic at Clitrofa - Centro Médico, Dentário e Cirúrgico, in Trofa - Portugal were screened. Ten patients scheduled for a bimaxillary osteotomy involving a combination of maxillary Le Fort I impaction procedure coupled with a sagittal split advancement of the mandible were selected to form the study group.

The patients have Magnetic Resonance Imaging (MRI), using the scan data obtained, together with the technique of 3D representation, changes in fibre orientation at the different jaw positions were evaluated with Anatomics ${ }^{\top M}$ Software. The landmarks considered for this study were: (a) the anterior angle from the long axis of masseter muscle versus angle between lower border of the zygomatic bone and the mastoid process, (b) the anterior angle from the long axis of the masseter muscle versus the mandibular plane.

The values were registered before surgery (T0) and 6 to 12 months after surgery (T1) and 1 week later (T2). The results have been measured by two different observers, according to the protocol jointly developed between the Eastman Dental Institute - University of London and the MRI Centre - Department of Radiology at John Radcliffe Hospital - University of Oxford. A combination of different parametric tests has been used to compare the different experimental variables. The experimental design devised for this study is depicted in Figure 2, comprising a combination of different examiners, surgical angles and times of measurement (pre- and post-operation).

\section{Comparison A - Testing the Differences between Examiners \\ ( $\mathrm{F}$ versus $\mathrm{N}$ )}

Research question: Are there any differences in the mean values of $\mathrm{P} 1$ and $\mathrm{P} 2$ angles measured by Examiner $\mathrm{F}$ and Examiner $\mathrm{N}$ in the same experimental conditions?

HO: There are no differences in the mean values of P1 and P2 angles measured by Examiner $\mathrm{F}$ and Examiner $\mathrm{N}$ in the same experimental conditions.

$\mathrm{H} 1$ : There are differences in the mean values of P1 and P2 angles measured by Examiner $\mathrm{F}$ and Examiner $\mathrm{N}$ in the same experimental conditions.

The statistical comparison between the examiners $\mathrm{F}$ and $\mathrm{N}$ regarding the measurement of "P1 Masseter Muscle/Zygomatic Bone/Process Mastoid Anterior Angle" and "P2 Masseter Muscle/ Mandibular Angle" of ten different patients was performed using a Paired Student's t-test for three different time moments of measurement (Time 0, Time 1 and Time 2).

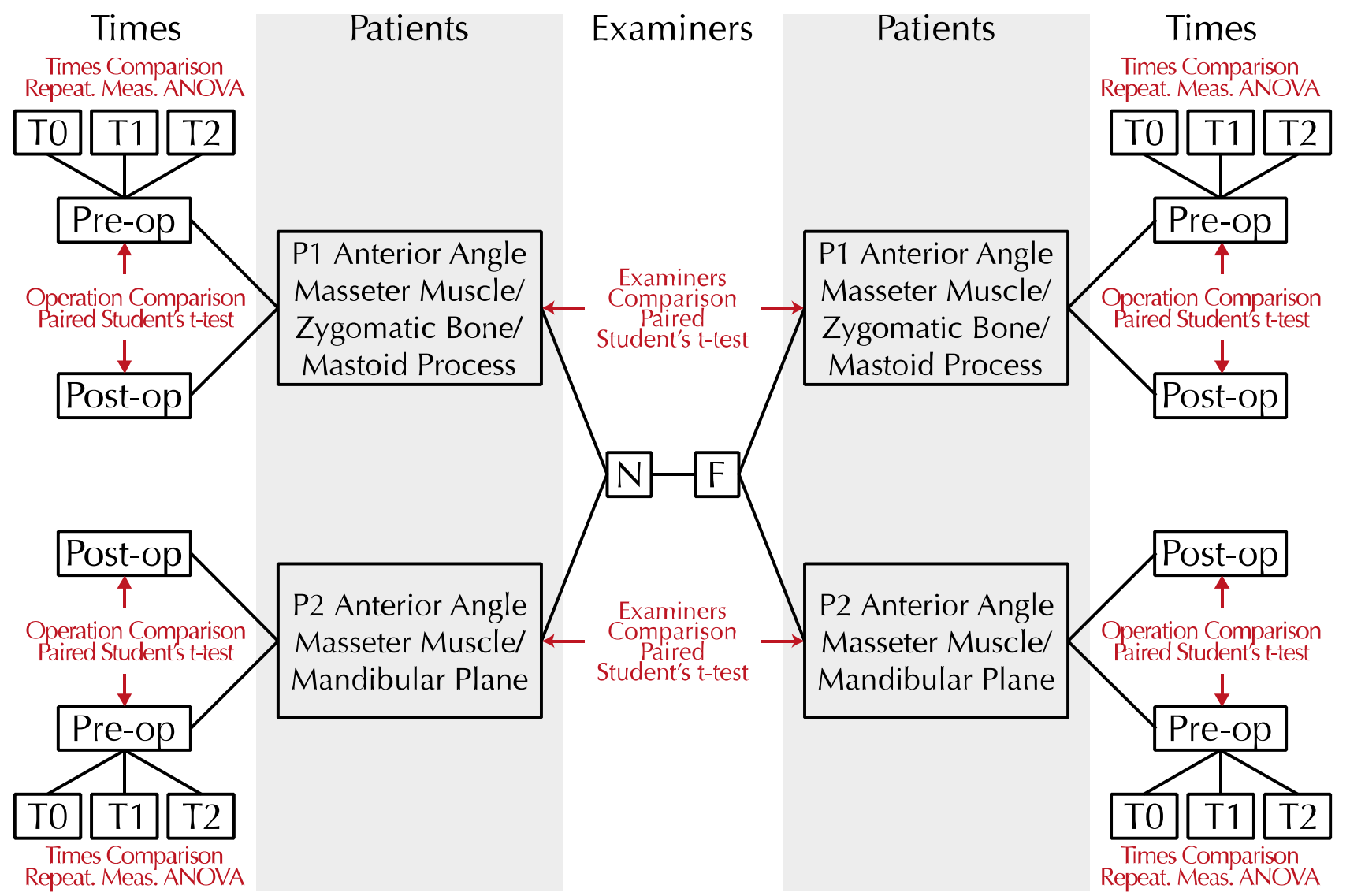

Figure 2. Experimental design used for assessing the biomodelling analysis. The study involved the contribution of two independent examiners ( $F$ and $N$ ), that measured the "P1 Masseter Muscle/Zygomatic Bone/Process Mastoid Anterior Angle" and the "P2 Masseter Muscle/Mandibular Angle" at two different times (pre- and post-operation) 


\section{Comparison B - Testing the Differences between Times (Time 0 versus Time 1 versus Time 2)}

Research question: Are there any differences in the mean values of $\mathrm{P} 1$ and $\mathrm{P} 2$ angles measured between moments Time 0 , Time 1 and Time 2 in the same experimental conditions?

$\mathrm{HO}$ : There are no differences in the mean values of P1 and P2 angles measured between moments Time 0 , Time 1 and Time 2 in the same experimental conditions.

$\mathrm{H} 1$ : There are differences in the mean values of $\mathrm{P} 1$ and $\mathrm{P} 2$ angles measured between moments Time 0 , Time 1 and Time 2 in the same experimental conditions.

The statistical comparison between the three time moments (Time 0, Time 1 and Time 2) regarding the measurement of "P1 Masseter Muscle/Zygomatic Bone/Process Mastoid Anterior Angle" and "P2 Masseter Muscle/Mandibular Angle" of ten different patients was performed using a Repeated Measure ANOVA for Examiner $\mathrm{F}$ and Examiner $\mathrm{N}$.
Table 1. Values of "P1 Masseter Muscle/Zygomatic Bone/Process Mastoid Anterior Angle" and "P2 Masseter Muscle/Mandibular Angle" of ten different patients observed prior to surgical operation ("pre-op"), at the different experimental conditions shown in Figure 2 .

\begin{tabular}{|l|l|l|l|}
\hline \multicolumn{1}{|c|}{ Variable } & Mean $\left({ }^{\circ}\right)$ & SD $\left({ }^{\circ}\right)$ & $\begin{array}{c}\text { Variance } \\
\left({ }^{\circ} \mathbf{\wedge}\right)\end{array}$ \\
\hline P1_F_T0 & 81,700 & 14,283 & 204,011 \\
\hline P1_F_T1 & 81,800 & 14,390 & 207,067 \\
\hline P1_F_T2 & 81,900 & 14,700 & 216,100 \\
\hline P1_N_T0 & 83,400 & 15,421 & 237,822 \\
\hline P1_N_T1 & 84,200 & 15,648 & 244,844 \\
\hline P1_N_T2 & 83,500 & 14,744 & 217,389 \\
\hline P2_F_T0 & 77,500 & 6,704 & 44,944 \\
\hline P2_F_T1 & 77,400 & 6,518 & 42,489 \\
\hline P2_F_T2 & 77,300 & 6,567 & 43,122 \\
\hline P2_N_T0 & 79,700 & 5,851 & 34,233 \\
\hline P2_N_T1 & 80,100 & 5,259 & 27,656 \\
\hline P2_N_T2 & 79,400 & 5,542 & 30,711 \\
\hline
\end{tabular}

\section{Comparison C - Testing the Differences between Operations \\ (Pre-op versus Post-op)}

Research question: Are there any differences in the mean values of P1 and P2 angles measured between prior ("pre-op") and after ("post-op") surgical intervention in the same experimental conditions?

H0: There are no differences in the mean values of P1 and P2 angles measured between "pre-op" and "post-op" moments in the same experimental conditions.

$\mathrm{H} 1$ : There are differences in the mean values of $\mathrm{P} 1$ and $\mathrm{P} 2$ angles measured between "pre-op" and "post-op" moments in the same experimental conditions.

The statistical comparison between pre-operative ("pre-op") and post-operative (post-op") values of "P1 Masseter Muscle/ Zygomatic Bone/Process Mastoid Anterior Angle" and "P2 Masseter Muscle/Mandibular Angle" of one selected patient observed by Examiners $\mathrm{F}$ and $\mathrm{N}$ was performed using a Paired Student's t-test.

\section{RESULTS}

Tables 1 and 2 present the experimental data for the measurement of P1 and P2 angles, showing low experimental variability, as can be assessed by SD and variance values.

Table 2. Values of "P1 Masseter Muscle/Zygomatic Bone/Process Mastoid Anterior Angle" and "P2 Masseter Muscle/Mandibular Angle" of a selected patient observed prior ("pre-op") and after ("post-op") the surgical operation, at the different experimental conditions shown in Figure 2 .

\begin{tabular}{|l|c|c|c|}
\hline \multicolumn{1}{|c}{ Variable } & \multicolumn{1}{c|}{ Mean $\left(^{\circ}\right)$} & \multicolumn{1}{c|}{ SD $\left(^{\circ}\right)$} & $\begin{array}{c}\text { Variance } \\
\left({ }^{\circ}{ }^{\circ} \mathbf{2}\right)\end{array}$ \\
\hline P1_F_Pre-op & 95,500 & 0,837 & 0,700 \\
\hline P1_N_Pre-op & 88,833 & 0,753 & 0,567 \\
\hline P2_F_Post-op & 71,333 & 0,516 & 0,267 \\
\hline $\begin{array}{l}\text { P2_N_Post- } \\
\text { op }\end{array}$ & 69,500 & 0,548 & 0,300 \\
\hline
\end{tabular}




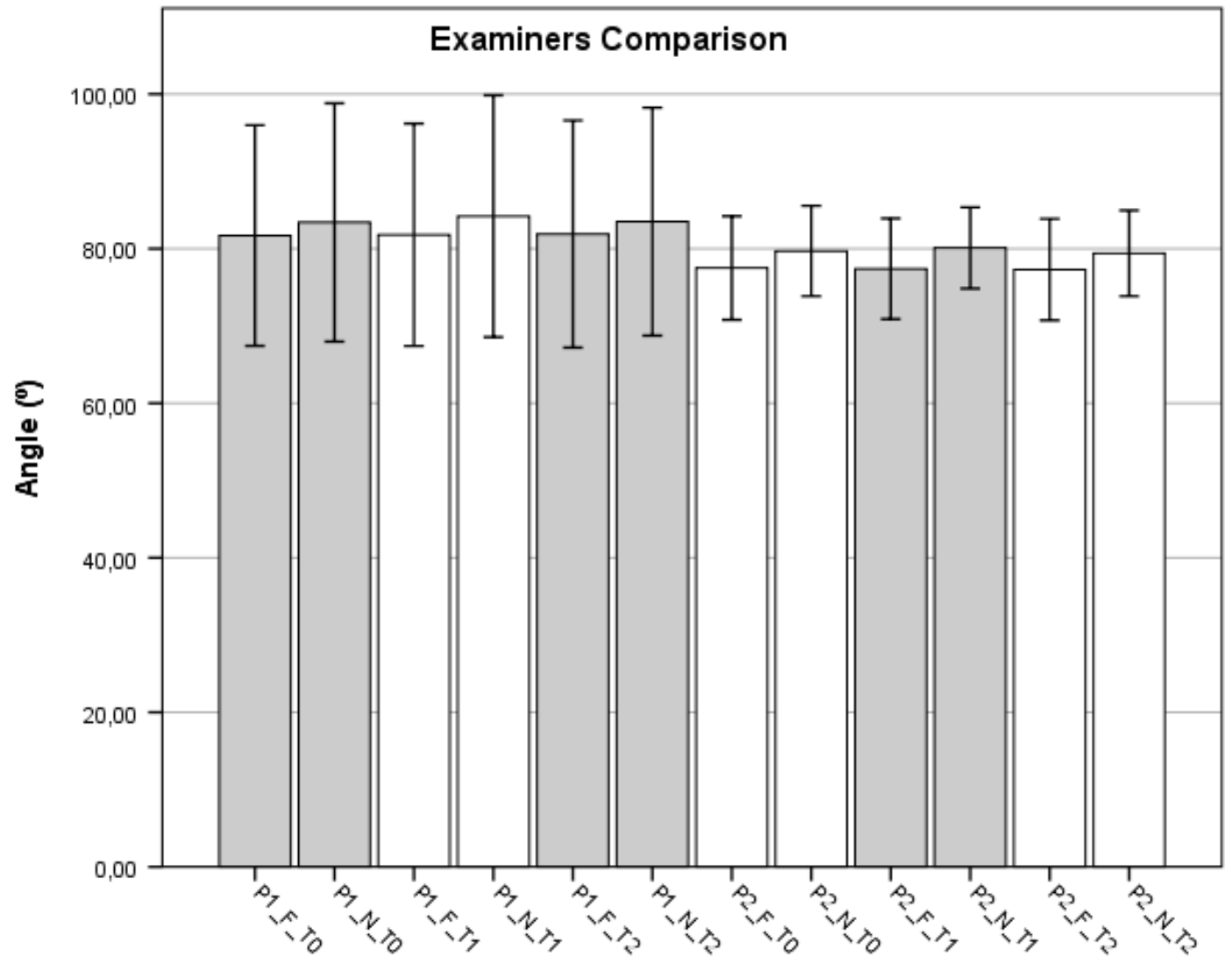

Figure 3. Mean values of "P1 Masseter Muscle/Zygomatic Bone/Process Mastoid Anterior Angle" and "P2 Masseter Muscle/Mandibular Angle" of ten different patients observed Examiner $F$ and Examiner $F$ at three different time moments (Time 0, Time 1 and Time 2).

Table 3. Statistical parameters obtained in the Paired Student's t-test for comparison of examiners F and N is regarding the measurement of "P1 Masseter Muscle/Zygomatic Bone/Process Mastoid Anterior Angle" and "P2 Masseter Muscle/Mandibular Angle" of ten different patients observed at three different time moments (Time 0, Time 1 and Time 2).

\begin{tabular}{|c|c|c|c|c|c|}
\hline Examiners Comparison & Mean Difference & $\begin{array}{l}\text { Standard } \\
\text { Deviation of } \\
\text { Differences }\end{array}$ & $\begin{array}{l}\text { Degrees of } \\
\text { Freedom (df) }\end{array}$ & $\begin{array}{c}\text { Test statistic } \\
\text { from Paired } \\
\text { t-test }\end{array}$ & $\begin{array}{l}\text { P-value from } \\
\text { Paired } t \text {-test }\end{array}$ \\
\hline $\begin{array}{l}\text { Examiner } \mathbf{F} \text { versus Examiner } \mathrm{N} \text {, } \\
\text { Time 0, P1 Angle }\end{array}$ & $-1,700$ & 2,497 & 9 & $-2,153$ & 0,060 \\
\hline $\begin{array}{l}\text { Examiner } \mathrm{F} \text { versus Examiner } \mathrm{N} \text {, } \\
\text { Time 1, P1 Angle }\end{array}$ & $-2,400$ & 2,221 & 9 & $-3,417$ & 0,008 \\
\hline $\begin{array}{l}\text { Examiner } \mathrm{F} \text { versus Examiner } \mathrm{N} \text {, } \\
\text { Time 2, P1 Angle }\end{array}$ & $-1,600$ & 1,838 & 9 & $-2,753$ & 0,022 \\
\hline $\begin{array}{l}\text { Examiner } \mathrm{F} \text { versus Examiner } \mathrm{N} \text {, } \\
\text { Time 0, P2 Angle }\end{array}$ & $-2,200$ & 3,910 & 9 & $-1,779$ & 0,109 \\
\hline $\begin{array}{l}\text { Examiner } \mathrm{F} \text { versus Examiner } \mathrm{N} \text {, } \\
\text { Time 1, P2 Angle }\end{array}$ & $-2,700$ & 3,529 & 9 & $-2,419$ & 0,039 \\
\hline $\begin{array}{l}\text { Examiner } \mathbf{F} \text { versus Examiner } \mathbf{N} \text {, } \\
\text { Time 2, P2 Angle }\end{array}$ & $-2,100$ & 3,213 & 9 & $-2,067$ & 0,069 \\
\hline
\end{tabular}

Statistical differences have been identified between Examiner $\mathrm{F}$ and Examiner $\mathrm{N}$ regarding the measurement of "P1 Masseter Muscle/Zygomatic Bone/Process Mastoid Anterior Angle" and "P2 Masseter Muscle/Mandibular Angle" of the ten patients analysed $(p<0,05)$. These discrepancies probably arise from small variations in the experimental methodology used by both examiners.

The standardization of the experimental protocol probably would reduce the differences detected. However, the maintenance of the same examiner in the evaluation of $\mathrm{P} 1$ and $\mathrm{P} 2$ angles for each patient would be the better approach to attain a high reproducibility. 


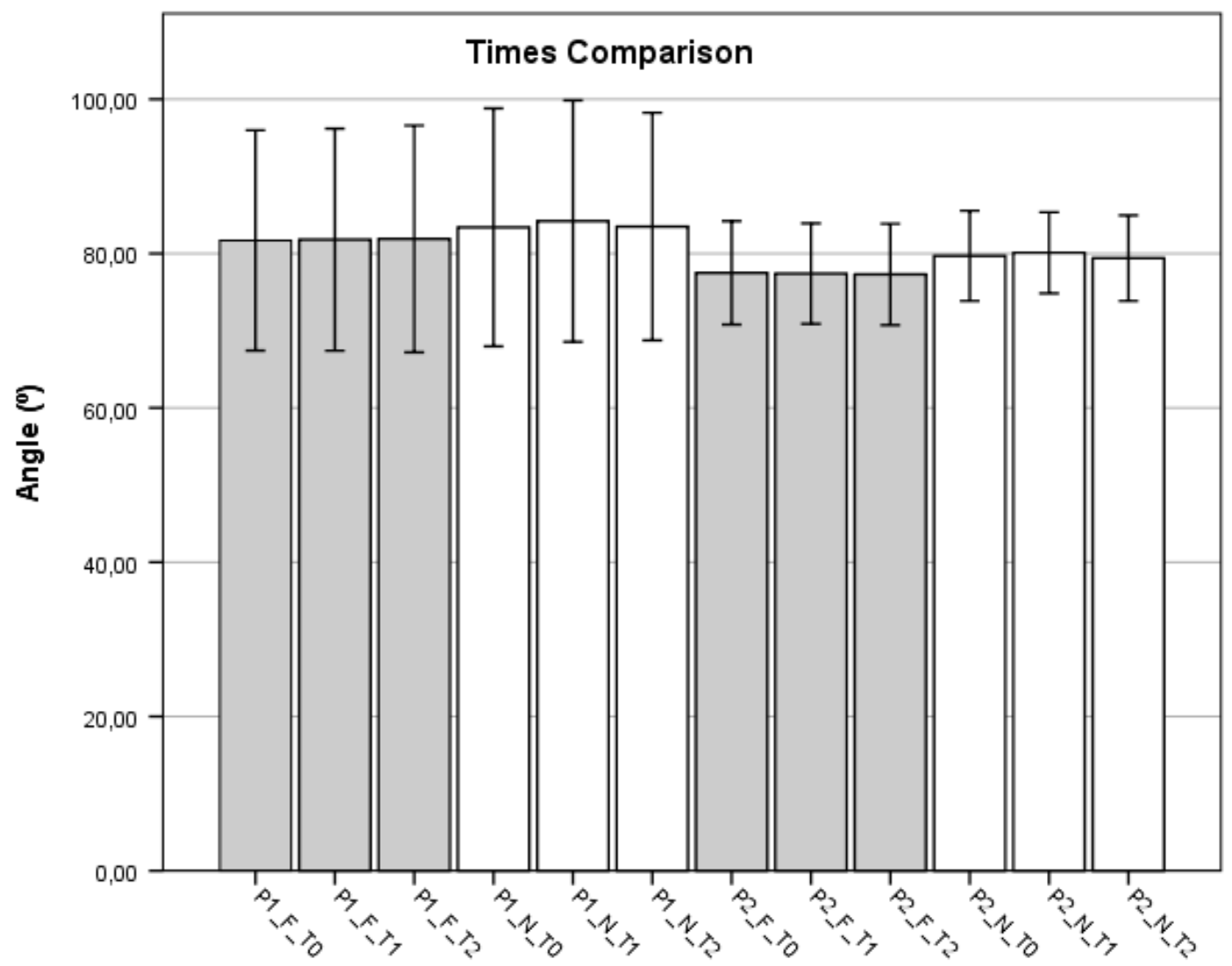

Figure 4. Mean values of "P1 Masseter Muscle/Zygomatic Bone/Process Mastoid Anterior Angle" and "P2 Masseter Muscle/Mandibular Angle" of ten different patients observed at three different time moments (Time 0, Time 1 and Time 2) by Examiner F and Examiner F.

Table 4. Statistical parameters obtained in the Repeated Measures ANOVA for the comparison of time moments (Time 0, Time 1 and Time 3) when measuring the "P1 Masseter Muscle/Zygomatic Bone/Process Mastoid Anterior Angle" and "P2 Masseter Muscle/Mandibular Angle" of ten different patients observed by Examiner F and Examiner F.

\begin{tabular}{|l|c|c|}
\hline \multicolumn{1}{|c|}{ Times Comparison } & \multicolumn{1}{c|}{$\begin{array}{c}\text { Degrees of } \\
\text { Freedom (df) }\end{array}$} & Test statistic (F) \\
\hline Time $\mathbf{0}$ vs Time $\mathbf{1}$ vs Time 2, Examiner F, P1 Angle & 2,18 & 0,403 \\
\hline Time $\mathbf{0}$ vs Time $\mathbf{1}$ vs Time 2, Examiner N, P1 Angle & 2,18 & 2,803 \\
\hline Time $\mathbf{0}$ vs Time 1 vs Time 2, Examiner F, P2 Angle & 2,18 & 0,474 \\
Time $\mathbf{0}$ vs Time 1 vs Time 2, Examiner N, P2 Angle & 2,18 & 2,043 \\
\hline
\end{tabular}

There are no significant differences in the mean P1 and P2 angles measured at Time 0 , Time 1 or Time 2, as long as the measurements are made by the same the same Examiner $(F$ or $\mathrm{N})$. All experiments reveal $\mathrm{p}$-values above the cut-off value of $0,05(p>0,05)$, which means that $\mathrm{H} 0$ proposition is valid. Thus, it is concluded the mean P1 and P2 angles $\left(^{\circ}\right)$ measured at different time frames are consistently the same, showing the high reproducibility of the measurements. 


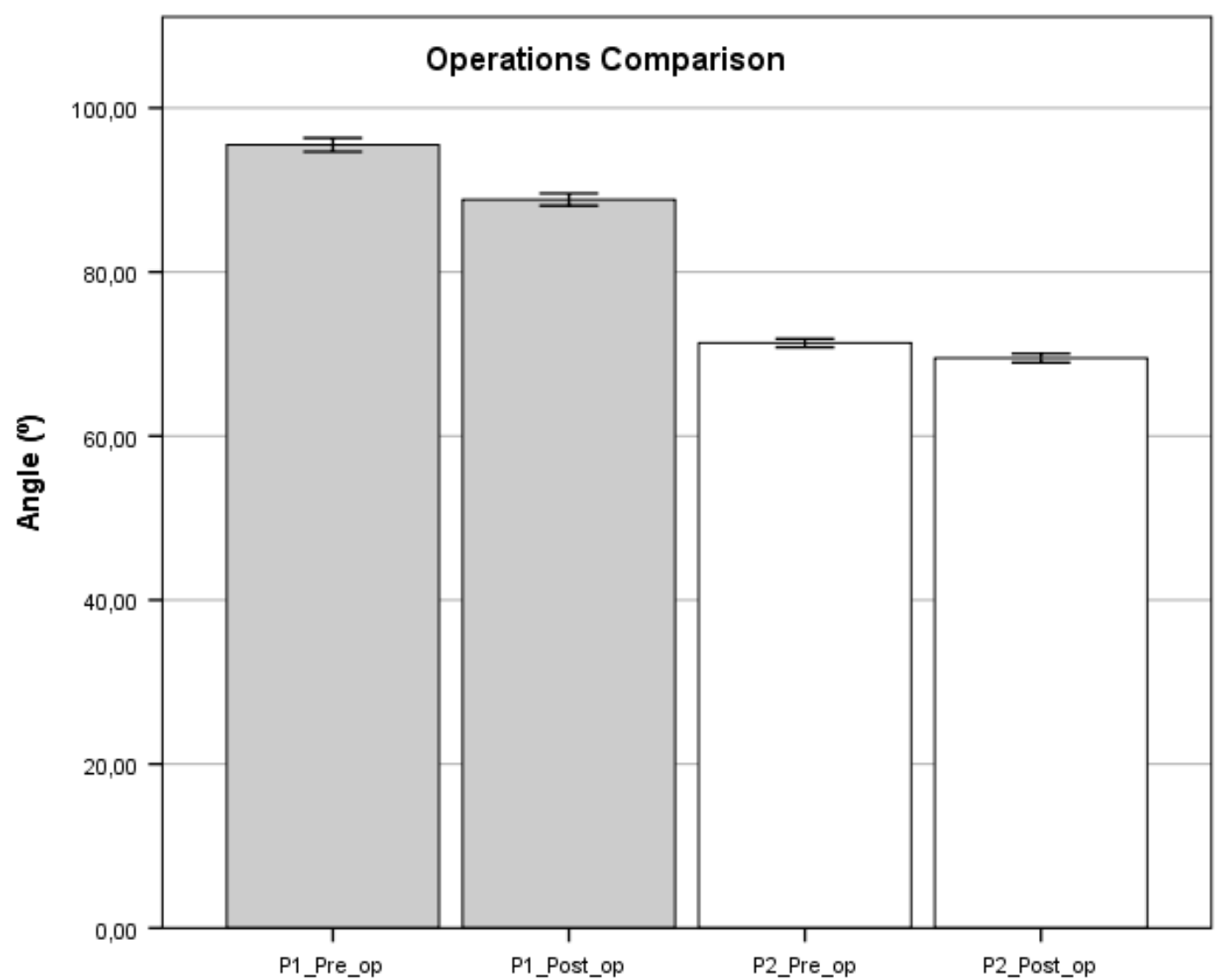

Figure 5. Mean values of "P1 Masseter Muscle/Zygomatic Bone/Process Mastoid Anterior Angle" and "P2 Masseter Muscle/Mandibular Angle" of a selected patient observed prior to surgical intervention ("pre-op") and after surgical intervention ("post-op").

Table 5. Statistical parameters obtained in the Paired Student's t-test for the comparison of "pre-op" and "post-op" values of "P1 Masseter Muscle/Zygomatic Bone/Process Mastoid Anterior Angle" and "P2 Masseter Muscle/Mandibular Angle" of a selected patient.

\begin{tabular}{|l|c|c|c|c|c|}
\hline \multicolumn{1}{|c}{ Examiners Comparison } & Mean Difference & $\begin{array}{c}\text { Standard } \\
\text { Deviation of } \\
\text { Differences }\end{array}$ & $\begin{array}{c}\text { Degrees of } \\
\text { Freedom (df) }\end{array}$ & $\begin{array}{c}\text { Test statistic } \\
\text { from Paired } \\
\text { t-test }\end{array}$ & $\begin{array}{c}\text { P-value from } \\
\text { Paired } t \text {-test }\end{array}$ \\
\hline Pre-op versus Post-op, P1 Angle & 6,66667 & 1,36626 & 5 & 11,952 & 0 \\
\hline Pre-op versus Post-op, P2 Angle & 1,83333 & 0,75277 & 5 & 5,966 & 0,002 \\
\hline
\end{tabular}

The Paired Student's t-test reveals the existence of statistical differences in the mean values of P1 and P2 angles before ("pre-op") and after ("post-op") the surgical intervention of the selected patient $(p<0,05)$.

These differences reveal the masseter muscle adaptation following orthognathic surgery in this study-case. The measurement of "P1 Masseter Muscle/Zygomatic Bone/Process Mastoid Anterior Angle" and "P2 Masseter Muscle/Mandibular Angle" can therefore be a valuable tool for controlling the reworking of masseter muscle upon orthognathic surgery.

\section{CONCLUSIONS}

Results show that the choice of the examiner may cause experimental variation on MRI data obtained for each patient, thus advising the use of standardized protocols to minimize these measurement differences. As for time reproducibility, the measurement of P1 and P2 angles has demonstrated to be consistent throughout the three time periods analysed, showing the high sensitivity of the MRI technique. Major statistical differences have been encountered, however, when comparing pre-op and post-op data for P1 and P2 angles, proving the applicability of MRI technique to evaluate masseter muscle adaptation following orthognathic surgery. These results indicate the high potential of MRI and biomodelling to predict the outcome of individual orthognathic surgeries, thus increasing the efficiency of these correction procedures.

\section{CONFLICT OF INTEREST}

The author declares that there is no conflict of interest regarding the publication of this article. 
12 | JSPIR

\section{REFERENCES}

1. D’Urso PS, Barker TM, Earwaker WJ, Bruce LJ, Atkinson RL, Lanigan MW, Arvier JF, Effeney DJ. Stereolithographic biomodelling in cranio-maxillofacial surgery: a prospective trial. J Craniomaxillofac Surg. 1999 Feb;27(1):30-7.

2. Opdebeeck H, Bell WH. The short face syndrome. Am J Orthod. 1978 May;73(5):499-511. doi: 10.1016/0002-9416(78)90240-3.

3. Schendel SA, Eisenfeld J, Bell WH, Epker BN, Mishelevich DJ. The long face syndrome: vertical maxillary excess. Am J Orthod. 1976 Oct;70(4):398-408.

4. Hunt N, Shah R, Sinanan A, Lewis M. Northcroft Memorial Lecture 2005: muscling in on malocclusions: current concepts on the role of muscles in the aetiology and treatment of malocclusion. J Orthod. 2006 Sep;33(3):187-97.

5. Hassfeld S, Mühling J. Computer assisted oral and maxillofacial surgery--a review and an assessment of technology. Int J Oral Maxillofac Surg. 2001 Feb;30(1):2-13.

6. Duarte F, Silva JN, Hopper C, Hunt N. Masseter Muscle Adaptation Following Orthognathic Surgery - MRI Analysis. Scientific Archives of Dental Sciences 2020; 3(7): 11-19.

7. Lewis MP, Machell JR, Hunt NP, Sinanan AC, Tippett HL. The extracellular matrix of muscle--implications for manipulation of the craniofacial musculature. Eur J Oral Sci. 2001 Aug;109(4):209-21.

8. Lewis MP, Tippett HL, Sinanan AC, Morgan MJ, Hunt NP. Gelatinase-B (matrix metalloproteinase-9; MMP-9) secretion is involved in the migratory phase of human and murine muscle cell cultures. J Muscle Res Cell Motil. 2000 Apr;21(3):223-33. 